\title{
Asma y alergia por el colorante carmín
}

\section{Asthma and allergy due to carmine dye}

\section{A.I. Tabar', S. Acero ${ }^{2}$, C. Arregui ${ }^{1}$, M. Urdánoz', S. Quirce ${ }^{3}$}

\section{RESUMEN}

El carmín de cochinilla o simplemente carmín E120) es un colorante rojo que se obtiene de las hembras desecadas del insecto Dactylopius coccus Costa (cochinilla)

Hemos evaluado la prevalencia de sensibilización y asma por carmín en una fábrica de colorantes naturales tras diagnosticar dos de sus trabajadores de asma ocupacional.

La incidencia acumulada de sensibilización y asma ocupacional por carmín en nuestra fábrica es de $48,1 \%$ y $18,5 \%$ respectivamente, datos que obligan a implantar medidas de prevención.

El asma ocupacional causado por inhalación de carmín debe considerarse como un ejemplo más de la capacidad que tienen ciertas partículas proteicas de artrópodos (en este caso las cochinillas) de actuar como aeroalergenos. El carmín debe añadirse a la lista de agentes capaces de producir asma ocupacional, cuyo mecanismo, según nuestros estudios, sería inmunológico mediado por anticuerpos IgE frente a diversos alergenos de alto peso molecular, que pueden variar de un paciente a otro. No obstante, dada la existencia de diferentes componentes en el carmín, no puede descartarse la posibilidad de que sustancias de bajo peso molecular, como el ácido carmínico, puedan actuar como haptenos.

Además, al tratarse de un colorante ampliamente utilizado como aditivo alimentario, como excipient farmacéutico y en la composición de numerosos cosméticos, no es de extrañar que puedan aparecer reacciones alérgicas tanto por su ingestión como por el contacto cutáneo directo.

Nos encontramos, ante un nuevo ejemplo de un alergeno que puede actuar tanto por vía inhalatoria alergeno que puede actuar tanto por vía inhalatoria como digestiva, dando lugar a un sindrome alergológico nes tanto de alergia respiratoria como alimentaria.

Palabras clave. Asma Ocupacional. Provocación inhalativa. Prevalencia. Incidencia acumulada. Colorante natural. Carmín. Cochinilla.

An. sis. sanit. Navar. 2003; 26 (Supl. 2): 65-73.

\section{ABSTRACT}

Cochineal carmine, or simply carmine (E120), is a red colouring that is obtained from the dried bodies of the female insect Dactylopius Coccus Costa (the cochineal insect).

We have evaluated the prevalence of sensitization and asthma caused by carmine in a factory using collowing the diagnosis of two workers with occupational asthma.

The accumulated incidence of sensitization and occupational asthma due to carmine in this factory are $48.1 \%$ and $18.5 \%$ respectively, figures that make the introduction of preventive measures obligatory.

Occupational asthma caused by inhaling carmine should be considered as a further example of the capacity of certain protein particles of arthropods (in this case cochineal insects) to act as aeroallergens. Carmine should be added to the list of agents capable of producing occupational asthma, whose mechanism according to our studies, would be immunological mediated by IgE antibodies in the face of diverse allergens of high molecular weight, which can vary from patient to patient. Nonetheless, given the existence of different components in carmine, it cannot be ruled out that substances of low molecular weight, such as carminic acid, might act as haptenes.

Besides, since we are dealing with a colouring that s widely used as a food additive, as a pharmaceutical excipient and in the composition of numerous cosmetics, it is not surprising that allergic reactions can appear both through ingestion and through direct cutaneous contact.

We find ourselves facing a new example of an allergen that can act through both inhalation and digestion, giving rise to an allergolical syndrome that digestion, giving rise to an allergolical syndrome that respiratory allergy and alimentary allergy.

Key words. Occupational asthma. Inhalative provocation. Prevalence. Accumulated incidence. Natura colouring. Carmine. Cochineal insect.
1. Servicio de Alergología. Hospital Virgen del Camino. Pamplona.

2. Sección de Alergia. Hospital № Sra de Sonsoles. Ávila

3. Servicio de Alergología. Fundación Jiménez Díaz. Madrid

\section{Correspondencia:}

Ana Isabel Tabar Purroy

Sección de Alergia. Hospital Virgen del Camino

CS Conde Oliveto.

Pza de la Paz s/n.

31002 PAMPLONA

Tfno: 948429308; Fax: 948429271 


\section{INTRODUCCIÓN}

La utilización de tintes y colorantes es probablemente tan antigua como la humanidad. Todas las civilizaciones han empleado diversas sustancias de procedencia orgánica o mineral, y más recientemente sintéticas, para colorear o decorar gran variedad de productos de su entorno, que van desde el propio cuerpo humano (cosméticos), a la vivienda, los utensilios, la ropa, los alimentos, etc. En el estudio de los colorantes están involucradas, por tanto, ciencias como la física, la química, la biología, la antropología, las artes plásticas, etc., implicando también a la medicina por las reacciones adversas que su utilización o consumo pueden producir en el ser humano.

Los colorantes se dividen de forma general en naturales y sintéticos, según su procedencia. Los tintes reactivos pertenecen al grupo de los colorantes sintéticos, y son los principales agentes implicados en patología ocupacional ${ }^{1}$. Los colorantes naturales también son ampliamente utilizados y a pesar de que son habitualmente bien tolerados, en los últimos años ha aparecido un número creciente de publicaciones sobre las reacciones alérgicas causadas por el colorante carmín. En este artículo revisaremos las reacciones alérgicas descritas con este colorante.

\section{CARMÍN DE COCHINILLA}

El carmín de cochinilla o simplemente carmín (E120) es un colorante rojo que se obtiene de las hembras desecadas del insecto Dactylopius coccus Costa (cochinilla). Este insecto pertenece a la familia Dactilopiidae, superfamilia Coccoidea, que está ampliamente distribuida y cuyos miembros tienen la peculiaridad de vivir en asociación o dependencia con una determinada especie vegetal, por lo que son considerados como plagas. El Dactylopius coccus Costa vive como parásito sobre cactus de los géneros Opuntia y Nopalea, especialmente Nopalea cochenillifera, de hábitat fundamentalmente en Sudamérica y Centroamérica. En España existen cultivos de estos insectos únicamente en las Islas Canarias. El proceso de obtención del carmín a partir de estos insectos consiste básicamente en extracción acuosa, filtrado, precipitación, secado, molido y esterilización, siendo finalmente envasado². El extracto de cochinilla es la solución concentrada que queda después de que se elimine el alcohol de un extracto acuosoalcohólico de insectos de cochinilla. El carmín es un polvo hidrosoluble compuesto por ácido carmínico en un sustrato de hidróxido de aluminio. Este polvo contiene aproximadamente un 50\% de ácido carmínico, sustancia que tiene un peso molecular de 492,4 Da y es el agente responsable del color, y también contiene alrededor de un $20 \%$ de material proteico proveniente de las cochinillas.

El carmín es uno de los colorantes rojos más apreciados, especialmente antes de la introducción de los colorantes artificiales, aunque todavía se utiliza ampliamente en las industrias alimentaria, cosmética (carmín rouge) y farmacéutica. El carmín y el extracto de cochinilla producen un color rojo rosado a magenta en los productos a los que se añade. Aunque estos colorantes se consideran generalmente inocuos para el ser humano, cada vez se describen con más frecuencia reacciones de hipersensibilidad frente a los mismos.

Por las excelentes propiedades tintoriales de este colorante, las cochinillas constituyeron uno de los principales objetos del comercio de Indias tras el descubrimiento de América, siendo sólo superado en valor e importancia por los metales nobles. El carmín se introdujo en Europa por los conquistadores españoles en 1519 tras descubrir su uso por los aztecas. Este tinte procedente del Nuevo Mundo encontró múltiples aplicaciones, incluyendo la tinción de textiles, pigmentos artísticos, alimentos y cosméticos. El colorante carmín se utiliza de varias maneras dependiendo de la coloración, solubilidad y características de estabilidad deseadas (Tabla 1). Puede utilizarse como un barniz insoluble para proporcionar el aspecto rojizo de la superficie externa del sucedáneo de carne de cangrejo (surimi) o en forma hidrosoluble en bebidas como el Campari, en zumos de frutas, productos lácteos como el yogur, helados o confituras. También puede añadirse a la bollería, 
Tabla 1. Utilización de colorantes de carmín y cochinilla ${ }^{3,17}$.

\begin{tabular}{lll}
\hline $\begin{array}{l}\text { Preparados de carmín } \\
\text { insolubles en agua }\end{array}$ & Preparados de carmín & $\begin{array}{l}\text { Extracto de cochinilla } \\
\text { Hidrosolubles }\end{array}$ \\
\hline Cosméticos & Productos cárnicos & Bebidas \\
Medicamentos & Embutidos & Yogures \\
Derivados lácteos & Helados & Helados \\
Bollería & Yogures & Conservas de frutas \\
Confituras & Conservas de frutas & Confites \\
& Bebidas, licores & Pudins \\
& Confituras, mermeladas & \\
& Caramelos y chicles & \\
& Bollería y galletas & \\
& Cosméticos & \\
& Medicamentos & \\
& & \\
& & \\
\hline
\end{tabular}

a ciertos cosméticos y a productos farmacéuticos o textiles. En la Unión Europea el carmín y el extracto de cochinilla deben etiquetarse E120 e internacionalmente se conocen como colorante rojo natural $\mathrm{n}^{\mathrm{o}} 4$. La OMS ha establecido un límite de consumo diario de $5 \mathrm{mg} / \mathrm{kg} / \mathrm{día}^{3}$.

\section{ALERGIA RESPIRATORIA}

La primera descripción de patología respiratoria causada por carmín corresponde a Burge y $\operatorname{col}^{4} \mathrm{y}$ hace referencia a dos pacientes que desarrollaron asma ocupacional por inhalación de carmín, uno de los cuales trabajaba en una fábrica de elaboración de carmín a partir de las cochinillas, y el otro era un empleado en una fábrica de cosméticos. Ambos pacientes presentaron reacciones asmáticas duales en la provocación con carmín, pero las pruebas cutáneas y la determinación de IgE específica (RAST) a carmín y cochinilla fueron negativas, encontrándose únicamente precipitinas frente a carmín en uno de los pacientes, por lo que no pudo confirmarse un mecanismo alérgico. En ambos pacientes la provocación oral con un extracto de cochinilla reprodujo los síntomas de broncoespasmo, y en uno de ellos provocó además dolor abdominal de tipo cólico.

En otro estudio, efectuado en una fábrica danesa de alimentos en la que se utilizaba el carmín como aditivo alimentario, se observó que a los pocos meses de introducir este colorante algunos trabajadores empezaron a presentar síntomas compatibles con alveolitis alérgica extrínseca ${ }^{5}$. Estos síntomas comenzaban entre 2 y 6 horas tras la exposición al polvo de carmín, remitiendo entre 12 y 36 horas tras finalizar la exposición. En tres de seis pacientes sintomáticos se encontraron precipitinas frente a una fracción de alto peso molecular del carmín, pero en estos pacientes no se realizaron pruebas de provocación bronquial. Posteriormente se ha confirmado el carmín como agente etiológico capaz de inducir asma ocupacional ${ }^{6,7} \mathrm{y}$ alveolitis alérgica extrínseca ${ }^{8}$.

En el Hospital Virgen del Camino de Pamplona se describió inicialmente el caso de un trabajador en una fábrica de producción de colorantes que presentaba asma ocupacional por inhalación del carmín ${ }^{9}$, encontrándose pruebas cutáneas en prick positivas y una respuesta dual en la provocación bronquial con este agente, aunque no se investigó la presencia de anticuerpos IgE específicos por métodos serológicos. Este paciente mejoró hasta quedar asintomático unos meses después de abandonar el trabajo. Posteriormente, en este mismo centro estudiamos a los 10 trabajadores de una fábrica de colorantes naturales alimentarlos ubicada en Tudela (Navarra), donde se producía carmín de cochinilla, annato (colorante amarillonaranja procedente de las semillas del árbol Bixa orellana) y cúrcuma (colorante amarillo-naranja que se obtiene del rizoma de Curcuma longa $V$. y se añade al curry). En una visita a dicha fábrica pudimos com- 


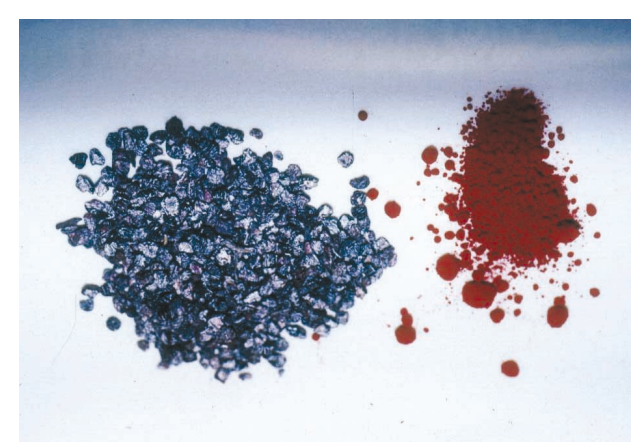

Figura 1. Cochinillas desecadas (izquierda) y carmín en polvo (derecha).

probar el ambiente densamente polvoriento que se producía durante la obtención del carmín, especialmente en la fase de molido del extracto desecado y durante el envasado, lo que obligaba a los trabajadores a llevar máscaras protectoras.

Además del paciente previamente descrito $^{9}$, que abandonó esta fábrica por presentar asma ocupacional, otro empleado, fumador y sin antecedentes de atopia, desarrolló síntomas de rinoconjuntivitis y asma a los pocos meses de comenzar a trabajar en la producción y envasado de colorantes, lo que relacionaba con la exposición al carmín $^{7}$. Este paciente mejoraba notablemente durante los fines de semana y en las bajas laborales hasta quedar asintomático. Los ocho trabajadores restantes no presentaban síntomas de asma. El registro seriado del PEF en el empleado con sintomatología ocupacional mostró la existencia de una variabilidad diaria en el PEF mayor del 20\% los días que procesaba el carmín. La provocación bronquial con metacolina demostró un alto grado de hiperreactividad bronquial inespecífica. Para el estudio alergológico preparamos extractos de carmín y de cochinilla al $10 \% \mathrm{p} / \mathrm{v}$ en solución salina tamponada con fosfato. En este paciente obtuvimos pruebas cutáneas en prick positivas con ambos extractos. Tras el acoplamiento de dichos extractos a discos de papel previamente activados con bromuro de cianógeno y siguiendo la técnica RAST, se obtuvieron niveles séricos elevados de anticuerpos IgE específicos para el carmín y la cochinilla. También se detectaron en este paciente anticuerpos de las subclases IgG1, IgG3 e
IgG4 frente al carmín, mediante técnica de ELISA. La relevancia clínica de la sensibilización a carmín se demostró mediante provocaciones bronquiales específicas, según la técnica de inhalación a volumen corriente durante 2 minutos de concentraciones crecientes, obteniéndose respuestas inmediatas con los extractos de carmín y cochinilla, pero no con el ácido carmínico. La provocación oral doble ciego hasta $100 \mathrm{mg}$ de carmín resultó negativa. En ninguno de los trabajadores expuestos asintomáticos encontramos IgE específica, aunque todos tenían niveles elevados de IgG específica frente al carmín, lo que indicaría la capacidad inmunógena de este agente tras la exposición por vía inhalatoria, sin otra repercusión clínica ${ }^{7}$. En un grupo de pacientes control todas estas pruebas fueron negativas. Por otro lado, ninguno de los trabajadores estudiados de esta fábrica, tanto sintomáticos como asintomáticos, tenían pruebas cutáneas positivas con los extractos de annato y cúrcuma al $10 \%$. En el paciente con asma ocupacional se realizó incluso una provocación bronquial con annato, con resultado negativo. Por tanto no existen indicios de sensibilización a estos colorantes en los pacientes estudiados.

En definitiva, todo apunta a que el paciente descrito presentaba asma ocupacional por sensibilización a carmín de cochinilla y que el mecanismo inmunopatogénico implicado se trata de una reacción de hipersensibilidad mediada por anticuerpos IgE específicos.

Seis años más tarde, al visitar de nuevo la fábrica, el personal había aumentado hasta 27 empleados y tres de ellos habían dejado el trabajo por síntomas respiratorios (Fig. 2). Con el fin de evaluar la sensibilización a carmín y la existencia de asma ocupacional, realizamos un nuevo protocolo que incluyó un cuestionario de asma tests cutáneos, test de inhalación con metacolina e IgE-dot ${ }^{10}$. Ante la sospecha de asma ocupacional también realizamos provocaciones inhalativas específicas y monitorización del PEF. Participaron en el estudio los 24 trabajadores en activo y otro más que recientemente había abandonado la fábrica por asma. El estudio se realizó en tres etapas (Fig. 3). En la primera, todos 


\begin{tabular}{|c|c|c|c|c|}
\hline & 1989 & 1992 & & 998 \\
\hline Empleados & 10 & 9 & 4 & 24 \\
\hline Sensibilizados a carmín & 1 & 2 & 1 & 10 \\
\hline \multirow[t]{2}{*}{ AO por carmín } & 1 & 1 & 1 & 2 \\
\hline & $\downarrow$ & $\downarrow$ & $\downarrow$ & \\
\hline Dejan el trabajo & $\stackrel{1}{\mathrm{OA}}$ & $\begin{array}{c}1 \\
\mathrm{OA}\end{array}$ & $\begin{array}{c}1 \\
\mathrm{OA}\end{array}$ & 0 \\
\hline
\end{tabular}

Figura 2. Esquema de la evolución de los empleados en la fábrica de colorantes.

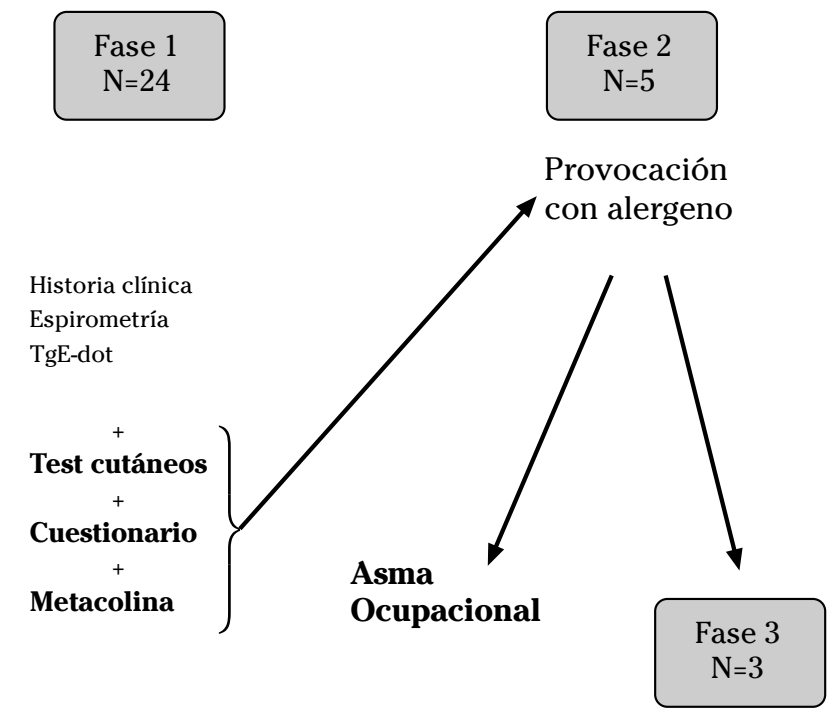

Registro PEP

Figura 3. Protocolo de estudio de los empleados en la fábrica de colorantes. 
los empleados completaron el cuestionario de síntomas de asma y a todos se les realizaron tests cutáneos con inhalantes habituales y ocupacionales (carmín, cochinilla, ácido carmínico, cúrcuma, annato y clorofila), espirometría basal y test de metacolina. Se obtuvo también una muestra de sangre para IgE-dot. En la segunda fase, a los empleados que cumplieron dos o más de los siguientes criterios: síntomas de asma en el lugar de trabajo (cuestionario positivo), prick positivo con al menos un alergeno ocupacional, o hiperreactividad bronquial, se les realizaron provocaciones bronquiales específicas. En la tercera etapa, los empleados que no reaccionaron a las provocaciones específicas efectuaron mediciones seriadas del PEF.

Los tests cutáneos con carmín, cochinilla y ácido carmínico fueron positivos en $6(26 \%), 7(29,2 \%)$ y 1 (4,2\%) trabajadores, respectivamente, y los realizados con cúrcuma, annato y clorofila, negativos. El análisis IgE-dot fue positivo en 4 de los 10 trabajadores con prick positivo y no se encontraron diferencias respecto a atopia y tabaco entre sujetos sensibilizados y los que no lo estaban. Uno de los trabajadores sensibilizados refería síntomas de rinitis exclusivamente. De los 5 que referían síntomas de asma en el trabajo, 2 tenían prick positivo y 4 hiperreactividad bronquial.

Realizamos provocación específica con los tres extractos positivos en prick en los seis empleados en activo que reunían criterios y en el trabajador que recientemente había dejado la fábrica. Obtuvimos respuesta asmática inmediata en dos y tardía en otros dos empleados con carmín y cochinilla. Los tres restantes realizaron registro de PEF durante 2 semanas en periodo laboral y otras dos sin contacto con dicho medio. Ningún registro fue sugestivo de asma ocupacional.

Por tanto, la prevalencia de sensibilización y de asma por carmín debemos situarla en 41,6 y $8,3 \%$. Sí incluyéramos los tres trabajadores que habían abandonado la fábrica hasta la fecha, tenemos que hablar de una incidencia acumulada de 48,1 y $18,5 \%$, cifras tan importantes como para hacer plantear la necesidad de implantar programas de prevención.
Acero y col $^{11}$, han descrito un paciente de 35 años, no atópico, que tras trabajar 4 años en un almacén de especias, comenzó a presentar rinoconjuntivitis y asma causadas por sensibilización al carmín mediada por IgE. Además este paciente experimentó rinoconjuntivitis y broncoespasmo minutos después de ingerir un caramelo de fresa que contenía el colorante E120. Los síntomas de este paciente se reprodujeron tras las pruebas de provocación inhalativa y oral con extractos de cochinilla y carmín, respectivamente ${ }^{11}$. Recientemente se ha descrito el asma por carmín en carniceros al elaborar hamburguesas, salchichas o embutidos con carmín ${ }^{12,13}$.

\section{ALERGIA ALIMENTARIA}

Se están describiendo cada vez con mayor frecuencia casos de reacciones alérgicas (urticaria, angioedema y anafilaxia) causadas por ingestión de alimentos coloreados por carmín. En muchos de estos casos se ha demostrado un mecanismo dependiente de IgE mediante pruebas cutáneas, liberación de histamina por los basófilos, determinaciones séricas de IgE específica a carmín y más recientemente con estudios de inmunodetección de aler genos (inmunoblot). En algún caso también se ha demostrado elevación de los niveles plasmáticos de triptasa durante las reacciones sistémicas ${ }^{3}$.

Puesto que el colorante carmín puede causar reacciones de hipersensibilidad inmediata, a veces muy graves, en concentraciones que se encuentran habitualmente en alimentos, bebidas y cosméticos, la posibilidad de alergia a este colorante debe sospecharse en pacientes que presenten urticaria recidivante, con o sin angioedema, o anafilaxia tras la ingestión o el uso de productos coloreados artificialmente.

Beaudouin y col $^{14}$ describieron en 1995 el primer caso de reacción anafiláctica causada por el consumo de un yogur coloreado con carmín en un paciente que tenía pruebas cutáneas positivas con un extracto de carmín y con el propio yogur. Estos autores estimaron que la cantidad de carmín ingerida por el paciente había sido de aproximadamente 1,3 mg. Posteriormente, 
Baldwin y $\operatorname{col}^{15}$ publicaron una paciente que había presentado un episodio de anafilaxia tras la ingestión de un polo helado que contenía carmín. Las pruebas cutáneas resultaron positivas con extracto de carmín y del polo implicado. Además demostraron un test de transferencia pasiva o P-K positivo con esta sustancia. En 1997 Wütrich y col ${ }^{16}$ publicaron un estudio en 5 mujeres de 25 a 43 años de edad que habían presentado reacciones anafilácticas tras la ingestión de vermut Campari (en cuya composición se encuentra el carmín) todas ellas con pruebas cutáneas e IgE sérica (mediante RAST) positivas para carmín. Solamente una de ellas tenía RAST positivo para un conjugado de ácido carmínico con albúmina sérica humana. Uno de estos pacientes ya había sido previamente publicado ${ }^{17}$.

DiCello y col $^{3}$ han descrito dos pacientes con episodios de urticaria, angioedema y anafilaxia tras la ingestión de productos que contenían carmín y han realizado una revisión bibliográfica sobre las reacciones adversas causadas por ingestión de carmín. En el primer caso se trata de una paciente no atópica que presentó una reacción anafiláctica tras la ingestión de yogur con sabor a fresa y plátano, en el que figuraba el carmín en la lista de ingredientes. Además refería varios episodios urticariales previos de causa desconocida. También había presentado prurito y edema periorbitario pocos minutos después de aplicarse una sombra de ojos con carmín. La segunda paciente era una enfermera que tenía una historia de múltiples episodios de angioedema facial y obstrucción nasal, en algún caso con anafilaxia, minutos después de tomar diversos alimentos o bebidas, tales como sucedáneo de cangrejo y licor Campari. En este caso también había presentado angioedema de párpados y urticaria facial tras aplicarse cosméticos con carmín. Las pruebas cutáneas en prick con extracto de carmín fueron positivas en ambas pacientes.

Recientemente Chung y $\mathrm{col}^{18}$ han descrito 3 mujeres de edades comprendidas entre 27 y 32 años que presentaron reacciones alérgicas (urticaria, angioedema y/o anafilaxia) por la ingestión de alimentos que contenían carmín (surimi, zumos, polos helados). En los 3 casos las pruebas cutáneas con carmín fueron positivas y en dos casos se confirmó la alergia al carmín mediante provocaciones orales.

\section{IDENTIFICACIÓN DE ALERGENOS}

Ya en los trabajos iniciales habíamos sugerido que los alergenos implicados en la alergia al carmín eran probablemente proteínas de las cochinillas que están presentes en su producto derivado, el carmín $^{7,19,20}$. En 1994 realizamos estudios de RAST e inhibición del RAST con cochinilla y carmín en 10 trabajadores de una fábrica donde se producía carmín ${ }^{7}$. Sólo en el trabajador que tenía asma ocupacional se encontró un RAST positivo al carmín, resultando negativo el RAST frente al conjugado ácido carmínico con albúmina sérica humana. Mediante estudios de inhibición del RAST con fracciones de carmín de diverso peso molecular pudimos demostrar que los alergenos principales tienen un peso molecular comprendido entre $10 \mathrm{y}$ $30 \mathrm{kDa}^{7}$.

En un estudio reciente investigamos los alergenos implicados en el asma ocupacional por carmín mediante inmunoblot $^{21}$. Para ello se separaron mediante electroforesis en gel de poliacrilamida (SDS-PAGE) las proteínas de los extractos de cochinilla (calentado y sin calentar) y de carmín. A continuación se realizó la inmunodetección de los componentes alergénicos utilizando suero de tres trabajadores con asma ocupacional por carmín, confirmados mediante provocaciones bronquiales específicas. Se visualizaron bandas proteicas de 30, 28 y $17 \mathrm{kDa}$ en el extracto de cochinilla cruda y otra banda de $50 \mathrm{kDa}$ en el extracto calentado. En el extracto de carmín se observaron dos bandas proteicas de aproximadamente 50 y 28 $\mathrm{kDa}$. Los componentes alergénicos que se detectaron con el suero de los pacientes fueron los siguientes: $17 \mathrm{kDa}$ en el extracto no calentado de cochinilla y $50 \mathrm{kDa}$ en el extracto calentado de cochinilla y un alergeno de $28 \mathrm{kDa}$ en el carmín.

En los tres casos de alergia alimentaria por ingestión de carmín descritos por Chung y col $^{18}$ también se realizaron estudios de inmunodetección de alergenos. 
Mediante SDS-PAGE se observaron varias bandas proteicas en el extracto de cochinilla, con pesos moleculares entre 23 y 88 $\mathrm{kDa}$. Existía una gran variabilidad individual en el reconocimiento de estas bandas proteicas con suero de las pacientes. La primera paciente reconocía componentes alergénicos de 38 y $50 \mathrm{kDa}$ en el carmín y la cochinilla, la segunda paciente reconocía un alergeno de $23 \mathrm{kDa}$ tanto en el carmín como la cochinilla y la tercera un alergeno de $88 \mathrm{kDa}$ únicamente en el extracto de cochinilla. Por lo general se reconocían más bandas alergénicas y con mayor intensidad en el extracto de cochinilla que en el extracto de carmín.

\section{DISCUSIÓN}

El asma ocupacional causada por inhalación de carmín debe considerarse como un ejemplo más de la capacidad que tienen ciertas partículas proteicas de artrópodos (en este caso las cochinillas) de actuar como aeroalergenos $^{22}$. El carmín debe añadirse a la lista de agentes capaces de producir asma ocupacional, cuyo mecanismo, según nuestros estudios, sería inmunológico mediado por anticuerpos IgE frente a diversos alergenos de alto peso molecular, que pueden variar de un paciente a otro. No obstante, dada la existencia de diferentes componentes en el carmín, no puede descartarse la posibilidad de que sustancias de bajo peso molecular, como el ácido carmínico, puedan actuar como haptenos. Además, al tratarse de un colorante ampliamente utilizado como aditivo alimentario, como excipiente farmacéutico y en la composición de numerosos cosméti$\cos$, no es de extrañar que puedan aparecer reacciones alérgicas tanto por su ingestión como por el contacto cutáneo directo. Nos encontraríamos, pues, ante un nuevo ejemplo de un alergeno que puede actuar tanto por vía inhalatoria como digestiva, dando lugar a un síndrome alergológico que puede presentarse clínicamente con manifestaciones tanto de alergia respiratoria como alimentaria. El caso del carmín no debe considerarse único entre los colorantes naturales. Otros colorantes, como el annato, también poseen capacidad sensibilizante, aunque por el momento sólo se ha comprobado por vía digestiva ${ }^{23}$. Los colorantes naturales comparten su naturaleza orgánica, bien sea animal o vegetal, y tienen en su composición cierta cantidad de material proteico, por lo que podrían actuar como antígenos potenciales. Si los hábitos socioculturales de los países desarrollados siguen favoreciendo el consumo de los productos denominados "naturales", entre ellos los colorantes, puede ser previsible un aumento en las reacciones alérgicas, tanto ocupacionales como no ocupacionales, a estas sustancias.

\section{BIBLIOGRAFÍA}

1. Alanko K, Keskinen H, BJöRKstén F, OJAnen, S. Immediate-type hypersensitivity to reactive dyes. Clin Allergy 1978; 8: 25-31.

2. LLOYD AG. Extraction and chemistry of cochineal. Food Chem 1980; 5: 91-107.

3. DiCello MC, Myc A, BAKER JR, BALdWin JL. Anaphylaxis after ingestion of carmine colored foods: two case reports and a review of the literature. Allergy Asthma Proc 1999; 20: $377-382$.

4. Burge PS, O'Brien IM, Harries MG, Pepys J. Occupational asthma due to inhaled carmine. Clin Allergy 1979; 9: 185-189.

5. Christiansen ML, Ahlbom G, Frank W et al. Extrinsic allergic alveolitis caused by occupational inhalation of carmine (abstract). Eur J Respir Dis 1981; 62: 82-83.

6. Tenabene A, Bessot JC, Lenz D, KofferschmittKuBler MC, PAULI G. Asthme professionnel au carmin de cochenille. Arch Mal Prof 1987; 48: 569-571.

7. Quirce S, Cuevas M, Olaguibel JM, Tabar AI Occupational asthma and immunologic responses induced by inhaled carmine among employees at a factory making natural dyes. J Allergy Clin Immunol 1994; 93 : 44-52.

8. Dietemann-Molard A, Braun, JJ, Sohier B, Pauli G. Extrinsic allergic alveolitis secondary to carmine (letter). Lancet 1991; 338: 460.

9. Rodríguez A, García de la Cuesta C, Olaguibel JM, TABAR AI, SANTOS F. Occupational asthma due to inhaled carminic acid dye: case report (abstract): Clin Exp Allergy 1990; 20 (Suppl. 1): 43.

10. Tabar AI, Alvarez MJ, Acero S, García BE, EChEChiPÍA S, Olaguibel JM et al. Carmine (E120)-induced occupational asthma revisited. J Allergy Clin Inmunol (en prensa). 
11. Acero S, Tabar AI, Alvarez MJ, García BE OlaGuiBel JM, MONEO I. Occupational asthma and food allergy due to carmine. Allergy 1998; 53: 897-901.

12. Escudero C, Cuesta J, Fernández-Nieto M, DE Miguel J, Pastor C, DE las Heras $M$ et al Occupational asthma due to carmine in a butchery worker (abstract). Allergy 2002; 57 (Suppl. 73): 113.

13. Marco FM, Ferrer A, Sempere JM, Andreu C, SANTO PJ. Occupational asthma to carmine in a sausage factory (abstract). Allergy 2002; 57: $113-114$

14. Beaudouin E, Kanny G, Lambert H. Food anaphylaxis following ingestion of carmine. Ann Allergy Asthma Immunol 1995; 74: 427430 .

15. Baldwin JL, Chou AH, Solomon WR. Popsicleinduced anaphylaxis due to carmine dye allergy. Ann Allergy Asthma Immunol 1997; 79: 415-419.

16. WÜTRICH B, KäGI MK, STÜCKER W. Anaphylactic reactions to ingested carmine (E120). Allergy 1997; 52: 1133-1137.
17. KäGI MK, WÜTRICH B, JOHANSSON SGO. Campariorange anaphylaxis due to carmine allergy. Lancet 1994; 344: 60-61.

18. Chung K, BaKer JR, BaLdwin JL, Chou A. Identification of carmine allergens among three carmine allergy patients. Allergy 2001; 56: 73-77.

19. Quirce Gancedo S, Olaguibel Rivera JM, Tabar PURROY AI. Alergia respiratoria ocupacional por tintes y colorantes. Rev Esp Alergol Inmunol Clin 1993; 8: 119-124.

20. Quirce Gancedo S, Olaguibel Rivera JM, Tabar PURROY AI. Asma ocupacional por colorantes. En: Asma ocupacional. Editores: Losada Cosmes E, Hinojosa Macías M. JR Prous Editores, Barcelona 1995, pp: 321-328.

21. Lizaso MT, Moneo I, García BE, Acero S, Quirce $\mathrm{S}$, TABAR AI. Identification of allergens involved in occupational asthma due to carmine dye. Ann Allergy Asthma Immunol 2000; 84: 549-552.

22. BELLAS TE. Occupational inhalant allergy to arthropods. Clin Rev Allergy 1990; 8: 15-29.

23. Nish WA, Wishman BA, GoETz DW, Ramírez DA. Anaphylaxis to annato dye: a case report Ann Allergy 1991; 66: 129-131. 